\title{
EMISSION OF ACRIDINE AND ITS AGGREGATES ISOLATED IN THE NEON MATRIX*
}

\author{
J. Prochorow, B. Kozankiewicz, Bango Bango Dongo Gemi \\ AND O. MORAWSKI \\ Institute of Physics, Polish Academy of Sciences \\ Al. Lotników 32/46, 02-668 Warsaw, Poland \\ (Received July 23, 1998; in final form October 30, 1998)
}

\begin{abstract}
The results of experimental studies of fluorescence and phosphorescence of acridine in the low-temperature inert neon matrix, at $7 \mathrm{~K}$, are reported. It is found that the low-temperature inert matrix of neon affects the energy levels of acridine molecules very weakly even as compared. with nonpolar (aprotic) and non-reactive solvent (e.g. hexane) and that there are different sites for acridine molecules in the neon matrix. However, the observed fluorescence spectra are strongly dependent on the excitation wavelength and besides the different (monomer) sites other emitting species are also contributing to the observed fluorescence emission of acridine in the neon matrix. Clear-cut evidence of the formation of singlet excimers of acridine in the neon matrix demonstrates itself as a very broad and structureless fluorescence spectrum with a relatively large shift from the origin of monomer (site) fluorescence which is characterized by a very distinct vibrational structure. The phosphorescence emission was observed only for the monomers. The observed differences in the low-energy part of excitation spectra of phosphorescence and fluorescence are discussed in terms of the close-lying excited singlet states of $n, \pi^{*}$ and $\pi, \pi^{*}$ character (mixed by the vibronic coupling) and tentatively interpreted as due to the formation of resonance acridine dimers. Their fluorescence spectrum is slightly shifted toward lower energies from the origin of monomer (site) fluorescence.
\end{abstract}

PACS numbers: 31.50.+w, 33.50.-j

\section{Introduction}

The origins and nature of fluorescence emission and the photophysics of excited acridine (ACD) molecule have been occupying the experimental research effort for almost forty years [1]. Their sensitivity to the solvent and to the phase conditions seems to be exceptional even when compared with other molecules

*The results of this paper were initially presented at The Jabloniski Centennial Conference on Luminescence and Photophysics, July 23-27, 1998, Torun', Poland. 
of policyclic monoazines and aromatic carbonyl families of organic compounds. From the very beginning of these investigations there were a dispute and a controversy as to the ordering (or inversion) of the lowest excited singlet states of $\pi, \pi^{*}$ $\left({ }^{1} L_{a}\right.$ and ${ }^{1} L_{b}$ states) and of $n, \pi^{*}$ electronic origin in ACD molecule $[2,3]$ :

Fluorescence quantum yield and its decay time for ACD molecule in different solvents may vary from virtually zero (in a gas phase and aprotic, non-hydrogen-bonding and/or hydrocarbon solvents) to relatively high values of the order of $10^{-2}$ (in hydrogen-bonding solvents, i.e., alcohols, water, etc.). And similarly, decay time of fluorescence of ACD varies from several picoseconds in hexane to several nanoseconds in water (for a review of earlier experimental data see [4]). Such data might be considered as an indication of strong $n, \pi^{*}$ character of the lowest excited singlet state and in favor of the model of inversion of ${ }^{1}\left(n, \pi^{*}\right)$ and ${ }^{1}\left(\pi, \pi^{*}\right)$ states in protic solvents, with a change of radiative properties of the lowest emitting state as the main cause of solvent-dependent quantum yield and decay time of ACD fluorescence. However, the results of low-temperature (in glassy solutions [3] and in MMA or PMMA matrices [5] at $77 \mathrm{~K}$ ) investigations of ACD fluorescence were suggesting that in these media (as well as in other solutions) the ${ }^{1}\left(\pi, \pi^{*}\right)$ singlet is the lowest one and that the solvent-dependent changes of the quantum yield and of decay time of fluorescence are due to the changes of non-radiative properties (internal conversion and intersystem crossing relaxation channels) of the emitting state caused by the solvent-dependent separation between close-lying ${ }^{1}\left(n, \pi^{*}\right)$ and ${ }^{1}\left(\pi, \pi^{*}\right)$ states which leads to the variation of the magnitude of a vibronic coupling between them (a phenomenon known as the proximity effect [6]). Neither time-resolved fluorescence measurements $[4,7]$ nor the transient triplet-triplet absorption measurements [8-10] were able to provide clear-cut evidence in favor of one of the above-mentioned models and the photophysical behavior of excited ACD molecules in solutions still remains somewhat obscured.

In parallel to the investigations in liquid and in rigid (amorphous) solutions, the excited ACD molecule and its photophysics were studied in an ordered crystalline phase. Neat crystals of ACD can be obtained in five crystalline modifications which differ by the unit cell dimensions, the space groups and by the number of molecules in the unit cell. It was shown that the fluorescence bands (very broad even at low temperatures and practically without vibrational structure) of these different crystalline modifications differ in spectral position as well as in their temporal characteristics [11-13]. Studies of the temperature dependence of spectral and temporal characteristics of the fluorescence of ACD crystals led to the conclusions that it originates from ${ }^{1}\left(\pi \pi^{*}\right)$ excited state and that the observed changes are thermally activated. Since, however, phosphorescence emission of ACD in these crystals was not observed the non-radiative relaxation channels of excited state of ACD were not identified. In addition, in two crystalline modifications of ACD (known as ACD II and ACD III) the formation of excited complex - an excimer was postulated, on the grounds of the observed temperature changes of fluorescence and of known details of the crystal structure (i.e., existence of two different types of packing units of ACD molecules in those crystalline modifications [13]). 
It should be born in mind, however, that the crystalline phase is very specific from the point of view of electronic excitation, due to the fact of its delocalization over an entire crystal within the excitonic bands (of singlet and of triplet character). Furthermore, in any real crystal, traps for excitons are always present, and with a change of temperature they can be depopulated either thermally or by biexcitonic annihilative events (which may result in different types of delayed fluorescence in addition to prompt fluorescence and/or phosphorescence). For these reasons, very often the analysis of the kinetics of emission as well as the identifcation of the relaxation pathways of excited electronic states of a crystal, turn out to be difficult experimental and theoretical tasks and sometimes without univocal interpretation.

Since, the molecule of ACD is a $N$-heterocyclic analog of anthracene and very closely fits the size and geometry of this latter it can easily be embedded into various host crystalline lattices of organic molecules and readily forms chemically mixed crystals especially with policyclic aromatic hosts in which a true site substitution in host lattice takes place and only the substitutional disorder is expected on the replacement of host molecule by the ACD molecule [14]. In an idealized case and at low concentrations of ACD, such a host lattice might be treated as an "inert matrix" in which ACD molecules are isolated in an ordered fashion which could enable the studies of their intrinsic photophysical properties (much alike as under gas phase collision-free conditions). Several such crystalline host matrices of chemically mixed crystals with ACD have been employed, but the results of these studies show a very different and individual photophysical behavior of ACD molecules in different matrices. In such crystalline host matrices as biphenyl [15] and fluorene [16] the lowest excited singlet state may be of strong ${ }^{1}\left(n, \pi^{*}\right)$ character. On the other hand in crystalline host matrix of 2,3-dimethylnaphthalene (DMN) the lowest excited singlet state of ACD must be of ${ }^{1}\left(\pi, \pi^{*}\right)$ character as inferred from a relatively strong and weakly temperature-dependent fluorescence with a rather long decay time of $8 \mathrm{~ns}$ at $4.2 \mathrm{~K}$ [17]. In addition, in this case also a very strong phosphorescence of $A C D$ is observed with very moderate intensity changes (of $c a .20 \%$ ) within a wide temperature range (4.2-300 K). Photophysics of excited molecule of ACD in DMN crystalline matrix seems to be controlled by practically temperature-independent intersystem crossing to the triplet manifold. The most complex situation was found in the case of crystalline host matrix of anthracene, which might have been considered as the most natural matrix for ACD molecule, at least as characterized by the very wide range of solid solubility limit for these two molecules $[18,19]$. In the anthracene crystalline matrix, however, the energies of the lowest singlet states of the host and guest (ACD) are very close to each other and hence, the formation of an amalgamated singlet excitonic band with the monomer level of ACD lying inside the excitonic band of anthracene crystal should be considered [20]. In fact in such chemically mixed crystals, with a low concentration of $A C D$, the observed fluorescence exhibits all features of fluorescence originating from a wide distribution of shallow traps of anthracene origin (the so-called X traps) induced by the introduction of ACD molecules into the anthracene host lattice [21]. At higher concentrations of ACD in the host lattice, a direct exchange interaction between ACD molecules leads to the formation of res- 
onance pairs (dimers) and higher aggregates (trimers, tetramers, etc.). This gives rise to new levels, shifted relative to the monomer level - the new trap levels of ACD origin are generated below the singlet excitonic band. Since the depth of these traps is increasing with the size of resonance aggregates, i.e., with an increase in concentration of ACD in the host lattice, even at deep temperatures $(4.2 \mathrm{~K})$ the fluorescence spectra are shifting toward lower energies [21]. Under these conditions the photophysics of ACD is effectively dominated by the aggregation-controlled spacing between nearly degenerate ${ }^{1}\left(n, \pi^{*}\right)$ and ${ }^{1}\left(\pi, \pi^{*}\right)$ states of ACD, due to the different resonance splitting of both states [22].

It is very clear that the energy levels diagram of ACD molecule is very sensitive to and strongly dependent on the physical (and chemical) conditions of the environment and obviously its details (ordering of $n, \pi^{*}$ and $\pi, \pi^{*}$ configurations within the singlet and triplet manifolds) as well as the detailed relaxation pathway of excited states must be determined for any particular medium, either liquid or solid. So far, earlier reports on the formation of ACD complexes in rigid media $[3,11,13,21-23]$ have been confirmed independently $[24,25]$ only for the case of singlet excimers of ACD in crystalline phase (in crystalline modification II). A possibility of the formation of ACD excimers is inherently connected with the crystal structure and packing conditions which provide and assure a preferable and necessary pre-orientation of interacting ACD molecules. The same seems to be true for the case of the above-mentioned resonance dimers (and higher aggregates) of ACD in the crystalline host lattice of anthracene.

In this work we attempt to apply the low-temperature inert(nobel)-gas matrix-isolation techniques for investigations of the emission of ACD and its presumable aggregates (excimers and/or resonance dimers). This technique has never been used before for investigations of ACD and although we cannot expect such preferable pre-orientation conditions for the formation of molecular complexes of ACD like in crystalline matrices, it has other advantages connected with the inertness of the nobel gas, close packing of matrix and deep temperatures. When combined with the narrow-band laser excitations it should also provide a good spectral resolution of fluorescence excitation spectra.

\section{Experimental section}

Zone-refined acridine and neon gas were mixed in a chamber heated to a temperature of $c a .60^{\circ} \mathrm{C}$. The content of neon gas in the chamber was regulated by the high precision needle valve (Edwards LV10K). The matrix was deposited onto a sapphire window located inside an optical liquid-helium cryostate (KR-5 model designed and produced in the Institute of Physics, Tartu, Estonia). The window on a cold-finger with a close contact with the liquid helium container had a temperature of $7 \mathrm{~K}$ (measured by the silicon diode temperature sensor attached to the cold finger). Thickness of the matrix layer was controlled during the deposition (a typical deposition time $-1 \mathrm{~h}$ ) by monitoring oscillations of interference of the light (670 $\mathrm{nm}$ line from a diode laser), reflected from the surfaces of the window and of the deposited layer. An estimated typical thickness of matrix layer was $100 \mu \mathrm{m}$. 
The emission of ACD was excited, with the use of the Lambda Physik FL1001 dye laser (lasing range 350-377 $\mathrm{nm}$ was covered with the DMQ dye) pumped by a LPX100 excimer laser. The fluorescence and fluorescence excitation spectra were observed at the right angle in respect to the direction of exciting light by using a $0.25 \mathrm{~m}$ Jarrel-Ash monochromator equipped with an EMI 9659 photomultiplier (cooled to $-20^{\circ} \mathrm{C}$ ). For the emission detection a sampling technique with a Stanford Research SR250 boxcar averager was used. A signal from boxcar was collected and analyzed by a PC using a LightScan photon counter card, which also controls the operation of the laser and monochromator. The phosphorescence emission was measured with the use of a Parker type phosphoroscope (with the synchronization of the laser action and operation of the disc chopper). Decay times of phosphorescence were accumulated with the aid of a. Stanford Research SR430 multichannel scaler. A part of the emission measurements was performed with the use of a $0.67 \mathrm{~m}$ McPherson spectrograph (model 207) equipped with a complete InstaSpec $\mathrm{V}$ gated intensified CCD-system (Oriel).

\section{Experimental results}

The experiments carried out in a way described in the preceding section have shown that the luminescence emission of neon matrix (at $7 \mathrm{~K}$ ) containing $\mathrm{ACD}$ molecules is composed of two types of emissions which differ in their spectral ranges as well as in their temporal characteristics.

\subsection{Fluorescence and fluorescence excitation spectra}

Fluorescence of ACD molecules in the neon matrix (at $7 \mathrm{~K}$ ) is observed as a relatively low-intensity emission in the range of $\sim 27500-19500 \mathrm{~cm}^{-1}$. Although the fluorescence spectra in the neon matrix exhibit a very rich and much better resolved vibrational structure than in the case of liquid solutions or rigid matrices (i.e., low-temperature organic glasses, PMMA matrices, neat ACD crystals and crystalline host matrices), still they are of the type of broad molecular bands. They are also dependent on the wavelength of excitation and exhibit a wide range of overlap with the excitation spectra, thus suggesting that they are composed of the fluorescence originating from different emitting species existing in the neon matrix.

In Fig. 1 the fluorescence spectra excited with different excitation wavelengths are compared. Close inspection of Fig. 1 reveals several regularities in the changes of fluorescence spectra of ACD upon the change of excitation wavelength. They can be summarized as follows:

- the fluorescence spectrum consists of a series of bands, with the separation of their centers of gravity of $\sim 1400 \mathrm{~cm}^{-1}$ and each of these bands has an additional structure of $\sim 90$ to $\sim 220 \mathrm{~cm}^{-1}$ (cf. Fig. 1 spectra $a-c$ ); the observed vibrational frequencies are known either from IR or Raman spectra of ACD [26];

- features of vibrational structure are clearly seen in the higher energy (27000-22000 $\mathrm{cm}^{-1}$ ) part of the fluorescence spectrum and start to fade away below $\sim 22000 \mathrm{~cm}^{-1}$ (cf. Fig. 1 spectra $a-c$ );

- a general outlook and the structure of fluorescence spectra do not change upon the change of excitation from high energy excitation $\left(\sim 28100 \mathrm{~cm}^{-1}\right)$ down 
to $27050 \mathrm{~cm}^{-1}$ (cf. Fig. 1 spectra $a-b$ ) - the only change is the change of intensity distribution within the vibrational bands;

- when the excitation is tuned to still lower energies $\left(26811 \mathrm{~cm}^{-1}\right)$ vibrational bands in fluorescence spectrum shift as a whole toward lower energies (cf. Fig. 1 spectrum $b$ and $c$ );

- with a still lower excitation energy (at $c a .26600 \mathrm{~cm}^{-1}$ ) fluorescence spectrum exhibits a very pronounced change - it appears as a very broad band of excimer-like character with a maximum at $\sim 21500 \mathrm{~cm}^{-1}$ (spectrum $d$ in Fig. 1) with some traces (or leftovers) of the vibrational structure.

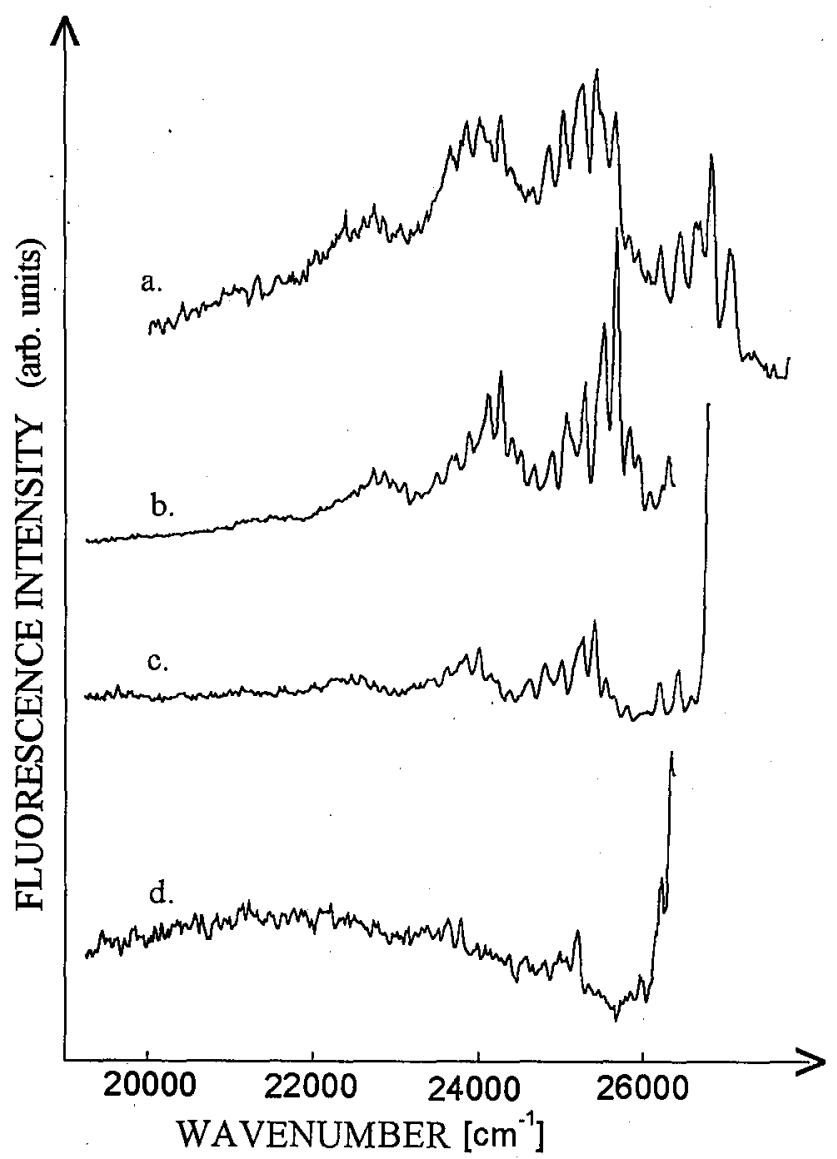

Fig. 1. Fluorescence spectra of acridine in the neon matrix (at $7 \mathrm{~K}$ ) observed upon excitation at: $28157 \mathrm{~cm}^{-1}(a), 27050 \mathrm{~cm}^{-1}(b), 26811 \mathrm{~cm}^{-1}(c)$ and $26600 \mathrm{~cm}^{-1}(d)$, respectively.

In Fig. 2 the fluorescence excitation spectra registered in the range of 26500$28500 \mathrm{~cm}^{-1}$ and monitored at different wavelengths within the fluorescence spectra (observation wavelengths) are collected. In general, as it can be seen from Fig. 2, 


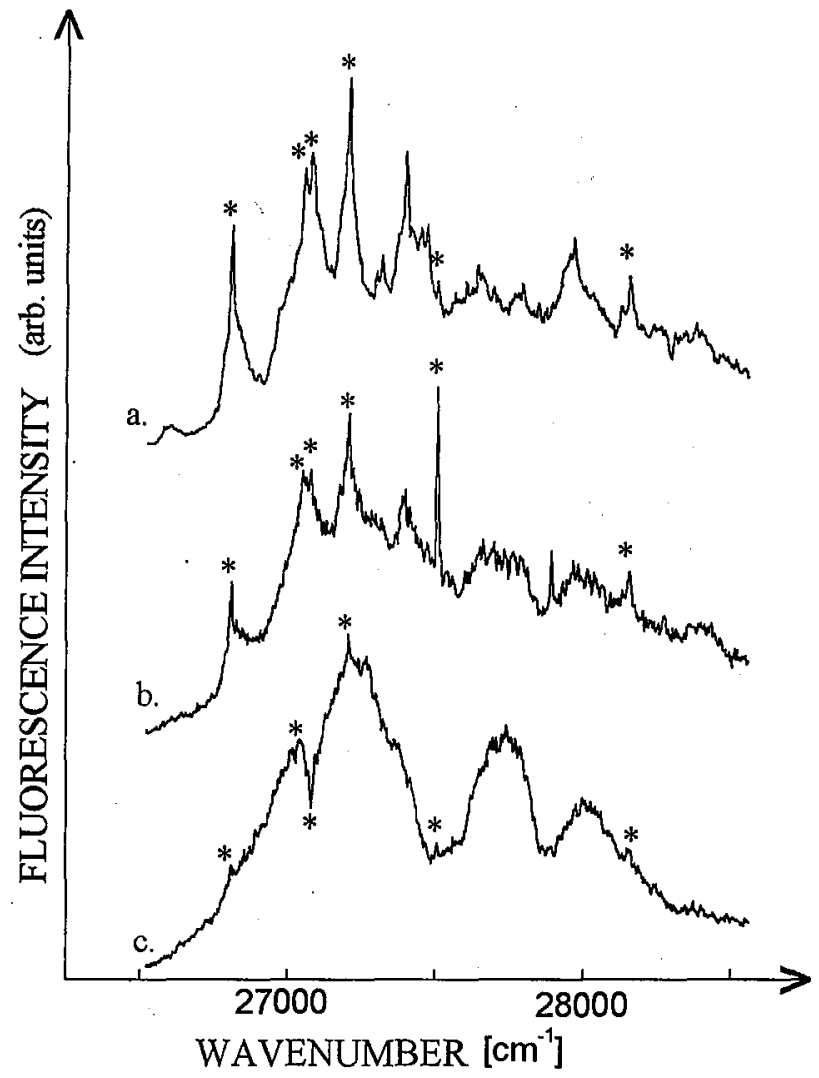

Fig. 2. Fluorescence excitation spectra of acridine in the neon matrix (at $7 \mathrm{~K}$ ) monitored at: $25200 \mathrm{~cm}^{-1}(a), 23630 \mathrm{~cm}^{1}(b)$ and $21500 \mathrm{~cm}^{-1}(c)$, respectively. The transitions which do not change upon a change of observation wavelength are marked with asterisks (see the text).

these spectra are composed of a relatively intense and wide background with a large variety of the vibrational bands. The spectra are strongly dependent on the wavelength of observation and the vibrational structure becomes less distinct with a decrease in the wavelength of observation. With the observation monitored at $\sim 21500 \mathrm{~cm}^{-1}$ (spectrum $c$ in Fig. 2), i.e., at the maximum of excimer-like fluorescence (cf. Fig. 1, spectrum $d$ ) practically only very diffuse vibrational bands are distinct. However, despite this strong. dependence of the fluorescence excitation spectra on the wavelength of observations, several relatively sharp transitions can always be seen at the same positions in all three spectra reproduced in Fig. 2, although their intensities are drastically changing from one spectrum to another (these were marked in Fig. 2 with asterisks and their wavenumbers are the following: 26811, 27050, 27075, 27202, 27506, 28133 and $28157 \mathrm{~cm}^{-1}$; with an accuracy of $\pm 5 \mathrm{~cm}^{-1}$ ).

Fluorescence decay times could not be measured with the available width of exciting laser pulse, i.e., they must be of the order or shorter than $5 \mathrm{~ns}$. 


\subsection{Phosphorescence and phosphorescence excitation spectra}

On the low-energy side of the fluorescence spectrum, a weak phosphorescence emission of the neon-matrix containing ACD is also observed (see Fig. 3a). The 0-0 band of the phosphorescence is positioned at $16016 \mathrm{~cm}^{-1}$ and thus it has a higher energy than the $0-0$ band of phosphorescence of ACD in chemically mixed crystal of ACD (host) with anthracene - $15691 \mathrm{~cm}^{-1}$ [25] and in crystalline host matrices of fluorene $-15749 \mathrm{~cm}^{-1}$ [23] and of dimethylnaphthalene $-15813 \mathrm{~cm}^{-1}$ [17] or in PMMA matrix (at $77 \mathrm{~K}$ ) $-15800-15900 \mathrm{~cm}^{-1}$ [5]. The observed phosphorescence spectrum contains such vibrational frequencies as $246,401,622,1170$ and $1271 \mathrm{~cm}^{-1}$, all of them have been previously observed in the phosphorescence

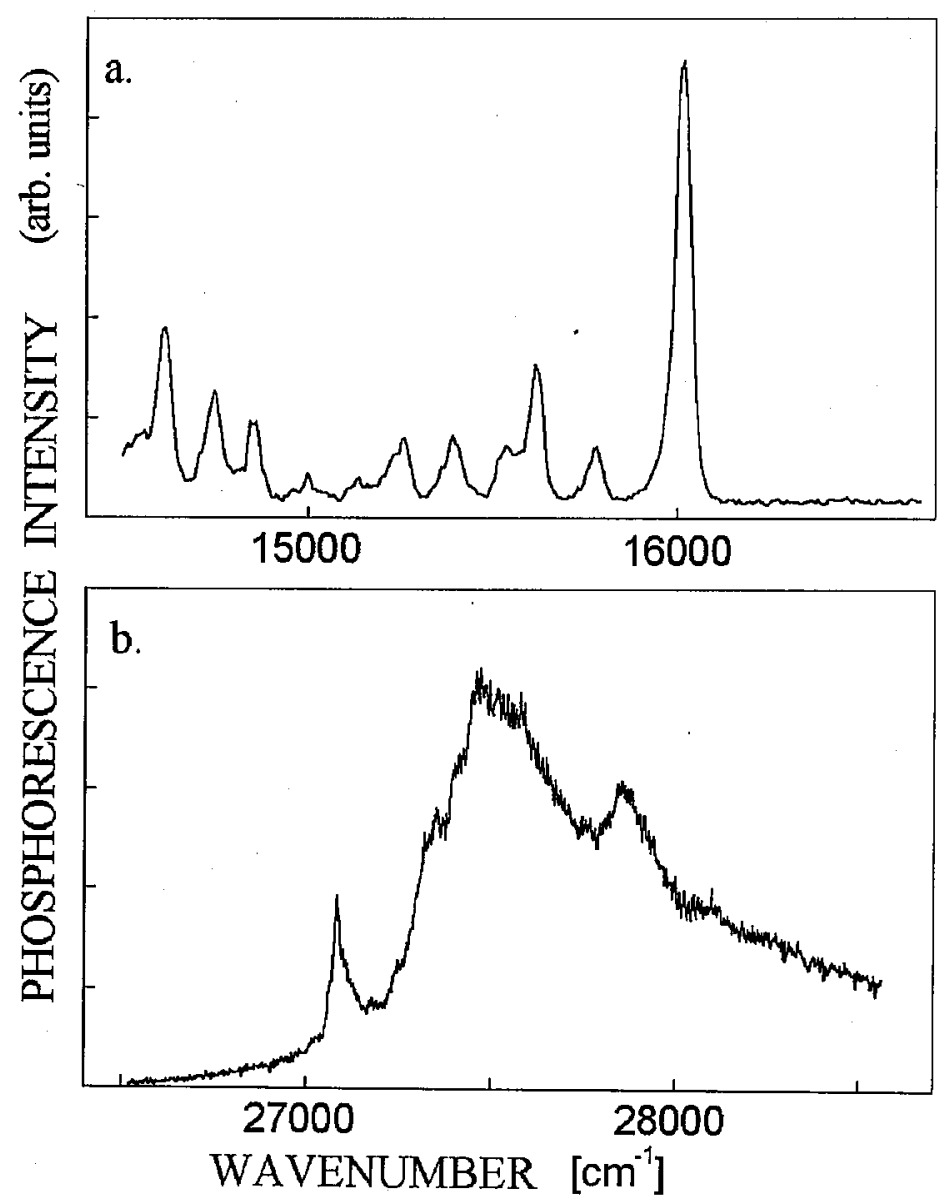

Fig. 3. Phosphorescence spectrum of acridine in the neon matrix (at $7 \mathrm{~K}$ ) registered for the range close to the $0-0$ band at $16016 \mathrm{~cm}^{-1}$ (a) and the phosphorescence excitation spectrum for this band (b). 
spectrum of ACD in the fluorene crystalline host matrix at $2.2 \mathrm{~K}$ [23], and also frequencies of 790,876 and $1410 \mathrm{~cm}^{-1}$ known from either IR or Raman spectra of ACD [26].

The decay times of phosphorescence measured for different wavelengths of observation are constant and equal to $25.5 \pm 1 \mathrm{~ms}$; they are also independent (within the error limit) of the excitation wavelength.

The phosphorescence excitation spectrum monitored at the $0-0$ band of the phosphorescence spectrum (i.e., at $16016 \mathrm{~cm}^{-1}$ ) is illustrated in Fig. 3b. This excitation spectrum is very broad and contains much less structural details as compared to the fluorescence excitation spectra (even if compared with the spectrum monitored for excimer-like fluorescence range). The only relatively sharp transition is that at $27075 \mathrm{~cm}^{-1}$ which is also observed (as a doublet of $25 \mathrm{~cm}^{-1}$ separation) in all three fluorescence excitation spectra (cf. Fig. 2). We notice also that the two other broad maxima of registered phosphorescence intensity, which are located at $c a .27550 \pm 30$ and $27860 \pm 20 \mathrm{~cm}^{-1}$, are corresponding to the minima of the registered fluorescence intensity in the fluorescence excitation spectra.

\section{Discussion and conclusions}

It is clear that the observed fluorescence and fluorescence excitation spectra are the combinations of several different band systems which might be connected with the existence of different sites (or conformers) of ACD molecules in the neon matrix. If we refer once again to the excitation spectra in Fig. 2 (cf. spectra $a$ and $b$ ) then we easily notice that such transitions as the above-mentioned lines at $27050 / 27075 \mathrm{~cm}^{-1}$ are of a doublet character (of $25 \mathrm{~cm}^{-1}$ separation). Thiey surely could be due to different sites of ACD molecules in the matrix. In this context it is notable that in the case of the excitation spectrum which is monitored in the range of excimer-like emission (spectrum $c$ in Fig. 2), the component of this doublet at $27075 \mathrm{~cm}^{-1}$ comes as a sharp "dip" of intensity of fluorescence (i.e., for this particular transition the emission is being reabsorbed). However, as seen in Fig. 2, in the range of $26811-28157 \mathrm{~cm}^{-1}$ there are several other sharp transitions (marked with asterisks in Fig. 2) which do not change in their spectral position in the excitation spectra (for different wavelengths of observation) and thus could presumably be ascribed as the origins of different sites of ACD in the neon matrix. The number and the structure of sites depend on the details of deposition and thermal treatment of the matrix as well as on the concentration of the molecules under investigations (in the case of anthracene in argon matrix as many as 11 different sites of anthracene were observed [27]). Since the observed bandwidths in the fluorescence excitation spectra are much wider than the spectral instrumental resolution (which is of $0.2 \mathrm{~cm}^{-1}$ ), these bands are inhomogeneously broadened and a part of such broadening may probably be due to the coupling with lattice phonons characteristic for a given site in the matrix. However, under conditions of our experiments we should take into account also a possibility of the formation of different complexes or aggregates of ACD, e.g., the formation of sandwich-like excimers as those confirmed for neat and mixed ACD crystals $[11,13,21,22]$, and the resonance aggregates such as those found in the crystalline host matrix of anthracene [24, 25]. The formation of such aggregations would 
result in additional broad bands or continuous background in the fluorescence and fluorescence excitation spectra.

Furthermore, in the case of ACD molecules the complexity of observed fluorescence excitation spectra can be increased by the fact of a close proximity of the $L_{a}{ }^{1}\left(\pi, \pi^{*}\right)$ and ${ }^{1}\left(n, \pi^{*}\right)$ singlet states. For instance, in the case of ACD in the crystalline host matrix of biphenyl, the vibronic origin of the ${ }^{1}\left(n, \pi^{*}\right)$ is lower only by $532 \mathrm{~cm}^{-1}$ with respect to the origin of ${ }^{1} L_{a}$ state, while a vibronic origin of ${ }^{1} L_{b}$ state is higher by $\sim 2100 \mathrm{~cm}^{-1}[15]$.

For all the above-mentioned reasons, the identification of different sites of ACD in the neon matrix and their origins must be left for a more precise high-resolution spectroscopic analysis of both the fluorescence and fluorescence excitation spectra in a function of ACD concentration in the matrix and accompanied by the vibrational analysis and exact assignment of all the lines in the observed band systems.

It is quite clear, however, that these data confirm the formation of singlet excimers of ACD in rigid media. Though very weak, the excimer fluorescence spectrum is observed as a typical very broad and structureless band shifted toward lower energies as compared to the monomer fluorescence of ACD (cf. Fig. $1 d$ ). It has a maximum at $c a .21500 \mathrm{~cm}^{-1}$ and its halfwidths is $\sim 4500 \mathrm{~cm}^{-1}$; due to a very low intensity its temporal characteristic could not be determined in the present study. These experimental data are providing a solid base for modelling of the optimized structures of ACD excimers and of their potential energy curves in the ground and excited states, in the way analogous to the modelling of structures and kinetics of the emission of intermolecular exciplexes [28,29].

The differences observed in the excitation spectra of fluorescence and of phosphorescence are providing some hints concerning the ordering and the nature of the lowest excited singlet and triplet states of ACD in the neon matrix. As mentioned earlier the fluorescence of ACD is weak and short-lived and thus it cannot be considered as of either pure $\pi, \pi^{*}$ or $n, \pi^{*}$ origin. As a matter of fact in the case of close proximity and strong interaction (and/or mixing) between ${ }^{1}\left(n, \pi^{*}\right)$ and ${ }^{1}\left(\pi, \pi^{*}\right)$ states it is more semantics than reality to describe these states in terms of pure zeroth-order (non-interacting) electronic configurations. With this remark in mind, we may tentatively ascribed the $27050 \mathrm{~cm}^{-1}$ transition as the $0-0$ transition of ACD molecule (from the lowest-energy neon-matrix site). Such an assignment locates the first excited singlet state (of mixed electronic character) of ACD molecule in the neon matrix at $27050 \mathrm{~cm}^{-1}$, i.e., at the energy of $c a .300-400 \mathrm{~cm}^{-1}$ higher than the energy of its $S_{1}$ (presumably of $n, \pi^{*}$ character) state in $n$-hexane [4] and of $\sim 1000 \mathrm{~cm}^{-1}$ higher than in such matrices as low-temperature PMMA glass [5] or crystalline matrix of DMN [17]. It is known that the third excited triplet state $T_{3}$ of $\pi, \pi^{*}$ character is located in the range of $26000-26500 \mathrm{~cm}^{-1}$ and that its position within this range is almost solvent-independent $[5,13,17,30]$. With the mixed character of $S_{1}$ state this would facilitate $S_{1} \leadsto T_{3}$ intersystem crossing, which followed by the internal conversion within the triplet manifold would result in the observation of measurable phosphorescence.

The transition at $26811 \mathrm{~cm}^{-1}$ which is observed in the fluorescence excitation spectra (cf. Fig. 2) also leads to the observation of ACD fluorescence (with 
the shift of $c a .240-250 \mathrm{~cm}^{-1}$ as compared to the fluorescence excited within the $27050 \mathrm{~cm}^{-1}$ band) and thus it could also be ascribed as the origin of another site of ACD in the neon matrix. However, this transition does not appear in the phosphorescence excitation spectrum, although, on the grounds of the same argumentation as presented just above, one might expect that the observation of phosphorescence emission would be equally or even more favorable, which is not the case. The reason of the decrease in efficiency of the $S_{1} \sim T_{3}$ intersystem crossing upon a rather slight change of the energy of $S_{1}$ can only be connected with the change of the nature of emitting species (with an effective change of the electronic character of $S_{1}$ state). This would imply that in this case the initially emitting $S_{1}$ state is (mostly) of $\pi, \pi^{*}$ character - in such case according to the El-Sayed's rule [31], the intersystem crossing between $S_{1}\left(\pi, \pi^{*}\right)$ and $T_{3}\left(\pi, \pi^{*}\right)$ would be less efficient than in the previous case. Such a change of the character of $S_{1}$ excited state can be due to the formation of resonance dimers and different magnitude of the resonance splitting for the monomer levels of different electronic character as observed in the case of chemically mixed crystals of ACD and anthracene [21,22]. The suggestion of the assignment of an absorption band at $26811 \mathrm{~cm}^{-1}$ to the formation of (resonance) dimers of ACD is, however, entirely based on the observed differences in the phosphorescence excitation spectra and thus would still require more clear-cut experimental evidence either from direct time-resolved measurements or from combined concentration-annealing steady-state fluorescence experiments. Both types of experiments are now in progress.

\section{Acknowledgments}

This work was performed in the main part within the framework of financial support of the Committee for Scientific Research under Project No. 2 P03B 08411.

We are also highly indebted to the Foundation for Polish Science for financing (within the MOLTEK Programm) the purchase of spectral detection system consisting of Model $207 \mathrm{McPherson}$ spectrograph equipped with a complete InstaSpec $\mathrm{V}$ gated intensified CCD-system.

\section{References}

[1] E.J. Bowen, J. Sahu, J. Chem. Soc. 3716 (1958).

[2] S.P. McGlynn, J. Chem. Phys. 37, 1825 (1962).

[3] S.J. Ladner, R. Becker, J. Phys. Chem. 67, 2481 (1963).

[4] L.A. Diverdi, M.R. Topp, J. Phys. Chem. 83, 3477 (1984).

[5] K. Kasama, K. Kikuchi, K. Uji-Ie, S. Yamamoto, H. Kokubun, J. Phys. Chem. 86, 4733 (1982).

[6] E.C. Lim, J. Phys. Chem. 90, 6670 (1986).

[7] S.L. Shapiro, K.R. Winn, J. Chem. Phys. 73, 1469 (1980).

[8] V. Sundstrom, P.M. Rentzepis, E.C. Lim, J. Chem. Phys. 66, 4287 (1977).

[9] L.J. Noe, E.O. Degenkolb, P.M. Rentzepis, J. Chem. Phys. 60, 4435 (1978).

[10] W.S. Struve, J.H. Hedstrom, C.G. Morgante, A.K. Jameson, E.C. Lim, J. Chem. Phys. 78, 7006 (1983). 
[11] B.P. Clarke, J.M. Thomas, J.O. Williams, Chem. Phys. Lett. 35, 251 (1975).

[12] J.O. Williams, B.P. Clarke, M.J. Shaw, Chem. Phys. Lett. 39, 142 (1976).

[13] J.O. Williams, B.P. Clarke, J. Chem. Soc. Faraday Trans. II 73, 514 (1977).

[14] A.I. Kitaigorodskii, Mixed Crystals, Spinger, Berlin 1983.

[15] D.L. Narva, D.S. McClure, Chem. Phys. 56, 167 (1981).

[16] B. Prass, F. Fujara, F. Seiff, D. Stehlik, J. Lumin. 24/25, 483 (1981).

[17] O. Morawski, J. Prochorow, Chem. Phys. Lett. 242, 253 (1995).

[18] R.M. Miasnikova, A.I. Kitaigorodski, Kr ristallografiya 3, 160 (1958).

[19] M. Radomska, R. Radomski, K. Pigoń, Mol. Cryst. Liq. Cryst. 18, 75 (1975).

[20] J.O. Williams, B.P. Clarke, J. Chem. Soc. Faraday Trans. II 73, 1371 (1977).

[21] O. Morawski, B. Kozankiewicz, J. Prochorow, R. Radomski, Chem. Phys. Lett. 150, 307 (1988).

[22] O. Morawski, B. Kozankiewicz, J. Prochorow, R. Radomski, Chem. Phys. Lett. 173, 412 (1990).

[23] R. Furrer, J. Gromer, A. Kacher, M. Schwoerer, H.C. Wolf, Chem. Phys. 9, 445 (1975).

[24] O. Morawski, J. Prochorow, Mol. Cryst. Liq. Cryst. 230, 173 (1993).

[25] O. Morawski, J. Prochorow, Acta Phys. Pol. A 88, 469 (1995).

[26] B. Schrader, Raman/Infrared Atlas of Organic Compounds, 2nd ed., VCH Verlagsgesellschaft, Weinheim 1989 (entry no. I10-01).

[27] J. Wolf, G. Hohlneicher, Chem. Phys. 181, 185 (1994).

[28] I. Deperasińska, J. Prochorow, J. Mol. Struct. (Theochem) 343, 11 (1995).

[29] I. Deperasińska, J. Prochorow, J. Mol. Struct. 436/437, 585 (1997).

[30] N. Periasamy, Chem. Phys. Lett. 99, 322 (1983).

[31] M.A. El-Sayed, J. Chem. Phys. 36, 573 (1962); ibid. 38, 2834 (1963). 